\title{
Réactions d'une série monosomique de blé à la contamination des feuilles et des épis par deux souches de Septoria nodorum Berk. (= Leptos- phaeria nodorum Mull.)
}

\author{
Frantz RAPILlY, Philippe AURIAU $(*)$, Hervé RICHARD \& Camille DePATUREAUX (*) \\ avec la collaboration technique de Bruno Letarnec, Laurent COUdARD \\ I.N.R.A., Station de Pathologie Végétale, \\ (*) Station de Génétique et d'Amélioration des plantes, route de Saint-Cyr, F 78026 Versailles Cedex
}

Les résultats présentés dans cet article sont la comparaison des réponses à la contamination d'une série monosomique complète de blé inoculée avec 2 souches de Septoria nodorum Berk., l'une isolée de blé var. « Etoile de Choisy » l'autre de triticale var. «Clercal». Ils confirment que la résistance partielle foliaire est gouvernée par des systèmes polychromosomiques en partie liés pour la durée d'incubation (L.P.I.) et pour la vitesse d'extension des surfaces contagieuses (P. max). Les critères de tolérance que sont le poids de 1000 grains malades (P.M.G.M.) et l'intensité des symptômes sur épis (I.E.) sont apparemment indépendants entre eux et indépendants des paramètres qui permettent de juger de la résistance particlle foliaire. Les interactions hautement significatives mises en évidence entre les chromosomes impliqués dans ces divers éléments de la résistance et entre souches du parasite permettent de s'interroger sur les différences qui existent ou non entre résistance verticale et résistance horizontale ou partielle. Enfin, une méthodologie de sélection est proposée pour accroître la résistance des blés à la septoriose.

Mots clés additionnels : Triticum aestivum $L .$, blé, Septoriose, résistance partielle, résistance verticale, résistance horizontale, analyse monosomique, sélection.

\begin{abstract}
A complete series of $F_{2}$ monosomic wheat was inoculated at boot and heading stages with two strains of Septoria nodorum Berk. ( = Leptosphaeria nodorum Muller). Strain n ${ }^{\circ} 6$ was isolated from wheat cv. "Etoile de Choisy", and strain $\mathrm{n}^{\circ} 42$ from triticale cv. "Clercal". The strains had different levels of aggressiveness. The $F_{2}$ monosomics were compared with the $\mathrm{F}_{2}$ disomic for reaction to the two strains, plant by plant, for the following characters : two elements of partial resistance (incubation time or L.P.I., rate of lesion extension or P. max); two elements of tolerance (disease intensity on the heads or I.E., thousand-kernel weight of diseased plants or P.M.G.M.). Table I and figure I show the results obtained with the two strains. For L.P.I., the two strains gave the same results on $\mathrm{F}_{2} 2 \mathrm{~A}, 7 \mathrm{~A}, 1 \mathrm{~B}, 2 \mathrm{~B}, 4 \mathrm{~B}, 1 \mathrm{D}, 2 \mathrm{D}, 3 \mathrm{D}$; only $7 \mathrm{~A}$ and $2 \mathrm{~B}$ gave a resistant reaction. The $\mathrm{F}_{2} 4 \mathrm{~A}$, 5B, 7B gave opposite results between the strains. With P. max, the same result for the resistance was given only with $3 \mathrm{~A}$, and opposite results with the $\mathrm{F}_{2} 2 \mathrm{~B}, 3 \mathrm{~B}, 5 \mathrm{~B}$. For I.E. the resistance or susceptibility reactions were different with the strains. No concordance was found between characters of partial resistance and characters of tolerance, which were thus two different genetic systems. The relation between L.P.I. and P. max was only partial with strain 42. Statistical analysis showed interaction between $F_{2}$ monosomic and strains for L.P.I. (table 2), for P. $\max$ (table 3), for I.E. (table 4) and for P.M.G.M. (tables 5 and 7). All three genomes of wheat were implicated but $\mathrm{F}_{2} 5 \mathrm{~A}, 1 \mathrm{~B}, 4 \mathrm{~B}, 5 \mathrm{D}, 7 \mathrm{~B}$ did not participate in these interactions (table 6). These results on variation in the chromosomes implicated in partial resistance in relation to the origin of the pathogen are discussed in regard to the specificity of the relation in the case of aggressiveness. There may be a risk of appearance of specialized strains adapted to the level of partial resistance in the host. A breeding program for resistance to glume blotch should associate partial resistance of the leaves and tolerance of the head.
\end{abstract}

Additional key words : Triticum aestivum $L$., wheat, glume blotch, partial resistance, vertical resistance, horizontal resistance, monosomic analysis, breeding. 


\section{INTRODUCTION}

Dans deux publications précédentes (Auriau et al., 1988; RaPILLY et al., 1988) nous avons démontré par l'analyse de $F_{1}$ monosomiques et de leur descendance $F_{2}$ que 2 éléments de la résistance foliaire du blé à Septoria nodorum ont une hérédité polychromosomique. Ces résultats enregistrés avec une souche du parasite isolée de la variété "Etoile de Choisy", nous ont aussi démontré que les critères de tolérance utilisés par TR.OTTET \& MERIEN (1982) ont, eux aussi une hérédité polychromosomique mais dépendant de chromosomes différents de ceux qui gouvernent les résistances partielles du feuillage. Pour apprécier la généralisation possible de ces résultats et les compléter, nous avons étendu nos investigations à la comparaison des réponses, de cette série monosomique à la contamination, par 2 souches du parasite supposées représentatives de la variabilité de son pouvoir pathogène.

\section{MATÉRIEL ET MÉTHODES}

\section{A. Matériel végétal}

C'est le même que celui utilisé dans une étude précédente (RAPILly et al., 1988); rappelons qu'il correspond aux descendances $F_{2}$ d'une série complète de $F_{1}$ issue du croisement entre la série monosomique du cultivar «Courtot » et la lignée « L 22 » sélectionnée pour son haut niveau de résistance partielle foliaire.

Les 21 chromosomes du blé ont des caractéristiques que nous rappelons : ils sont originaires de 3 génomes : 7 chromosomes constituant le génome A proviennent de Triticum monococcum L. spp. boeticum; 7 autres formant le génome B ont leur origine dans la section sitopsis des Aegilops et enfin les 7 chromosomes donnant le génome D proviennent d'Aegilops squarrosa. Ces 21 chromosomes sont aussi regroupés en 7 groupes dits homéologues et dénommés I, II, III, ... VII. Le groupe homéologue I comprend les chromosomes 1A, 1B, 1D suivant la nomenclature proposée par CHAPMAN \& RILEY (1966).

\section{B. Matériel fongique}

A la souche 6, isolée du blé var. "Etoile de Choisy » utilisée dans les études précédentes, est comparée la souche $\mathbf{4 2}$ isolée de triticale cv. "Clercal ». L'agressivité relative de cette souche a déjà été décrite (SKAJENNIKOFF \& RAPILLY, 1985). Sa principale particularité est de provenir d'un hôte entièrement nouveau : le triticale, et nous avons montré que dans la population 6 , pouvaient être « prélevés » par le triticale «Clercal » des éléments pathogènes qui lui sont adaptés (données non publiées). Pour maintenir les caractéristiques originelles, au cours du temps, de ces souches, elles sont entretenues sur leurs hôtes d'origine.

\section{Dispositif expérimental et inoculations réalisées}

L'essai comportait, d'une part, 2 répétitions contaminées aux stades gonflement et épiaison avec la souche 6 , et d'autre part, une seule répétition contaminée aux mêmes stades, avec la souche 42 . La parcelle élémentaire était constituée d'une ligne de 20 plantes de la descendance $F_{2}$ d'une même $F_{1}$ monosomique. Chaque répétition comportait les $21 \mathrm{~F}_{2}$ monosomiques (dénommées dans le texte: $F_{2}$ mono. ou $F_{2} 1 A$, $F_{2} 2 A \ldots$ ), la $F_{2}$ disomique (dénommée $F_{2}$ di.) qui a servi de terme de comparaison et une ligne d'une variété très sensible pour vérifier la réussite des contaminations et permettre, avec la $F_{2}$ disomique, de juger de l'homogénéité de l'essai.

\section{Notations et comparaisons réalisées}

\section{Notations}

Ce sont les mêmes que celles qui ont été faites dans nos études précédentes (AURIAU et al., 1988), conduites plante à plante, elles portent sur :

\section{a) Deux paramètres de résistance partielle foliaire}

- La durée dincubation ou L.P.I.

- La vitesse d'extension des nécroses foliaires (P. max) élément principal pour refléter la vitesse d'extension de la surface contagieuse.

\section{b) Deux critères de tolérance}

La tolérance est le fait qu'une plante malade subisse pas ou peu de pertes (RAPILLY, 1984), ici la réduction du poids de 1000 grains. Les critères utilisés sont :

- l'intensité des symptômes sur épis (I.E.). Ces symptômes permettent de reconnaître que l'épi est malade;

- le poids de 1000 grains issus de plantes malades (P.M.G.M.) : l'absence de témoins sains ne permet pas de calculer le poids relatif des grains malades qui est le critère de tolérance habituellement utilisé pour cette maladie. Toutefois on peut comparer entre eux les P.M.G.M. obtenus pour chacune des $F_{2}$. Les rapports P.M.G.M. 42 autorisent aussi de juger des effets des souches pour chaque $F_{2}$.

\section{Comparaisons réalisées}

A partir, soit des notations individuelles (pour mettre en évidence des interactions), soit des notes moyennes calculées par ligne permettant de connaître les diverses p.p.d.s. (pour la souche 6), plusieurs comparaisons ont été réalisées par rapport à la $\mathrm{F}_{2}$ disomique. Pour la souche 42, du fait de l'absence de répétition, nous avons retenu comme valeurs des différentes p.p.d.s. celles calculées pour la souche 6 multipliées par $\sqrt{2}$.

\section{RÉSULTATS}

\section{A. Validité de l'essai}

Le test de $\chi^{2}$, appliqué aux réponses de la $F_{2}$ disomique et aux plantes de la variété sensible, indique que la 
répartition de celles-ci par classe de gravité pour les paramètres notés, ne diffère pas entre les répétitions et les souches inoculées. Ainsi, pour la L.P.I. le $\chi^{2}$ varie entre 0,4 et 1,2 N.S. avec 1 d.d.1. ; pour P. max il est non significatif $\left(\chi^{2}=4,98\right.$ avec 5 d.d.l.), il en est de même pour I.E. $\left(\chi^{2}=6,22\right.$ N.S. avec 4 d.d.l. $)$. Les différences constatées entre la $F_{2}$ disomique et les $F_{2}$ monosomiques ont donc pour origine la réponse de celles-ci aux souches avec lesquelles elles ont été inoculées.

\section{B. Paramètres de résistance partielle foliaire}

\section{Durée d'incubation (L.P.I.)}

Le tableau 1 récapitule les réponses moyennes, de chaque ligne $F_{2}$ mono. par rapport à la $F_{2}$ disomique, obtenues avec les 2 souches du parasite. Huit chromosomes permettent un allongement de la durée de la L.P.I. (réaction de résistance) pour la souche 6 mais 2 seulement, les $7 \mathrm{~A}$ et $2 \mathrm{~B}$ sont communs avec la souche 42 . Une réduction significative de la L.P.I. (donc l'augmentation de la sensibilité) est enregistrée pour les 2 souches avec les chromosomes $2 \mathrm{~A}$ et $1 \mathrm{~B}$, auxquels s'ajoutent le $6 \mathrm{D}$ pour la souche 6 et 9 autres chromosomes pour la souche 42 . Au total, sur les 21 chromosomes de la lignée « $\mathrm{L} 22$ », 8 donnent la même réponse aux 2 souches du parasite. Mais, pour les $\mathrm{F}_{2} 4 \mathrm{~A}, 5 \mathrm{~B}, 7 \mathrm{~B}$, des réponses opposées sont constatées suivant les isolats ; la figure 1 illustre l'importance de ces inversions.

L'interprétation des résultats obtenus plante à plante, permet de mettre en évidence des interactions hautement significatives entre $F_{2}$ mono. et souches (tabl. 2) tant au niveau des génomes, qu'au niveau des groupes homéologues. Le rapprochement de ce tableau avec la figure 1 indique que les chromosomes $3 \mathrm{~A}, 4 \mathrm{~A}, 5 \mathrm{~B}, 6 \mathrm{~B}$, $7 \mathrm{~B}, 4 \mathrm{D}$ et $6 \mathrm{D}$ sont particulièrement impliqués dans celles-ci.

\section{Vitesse d'extension des nécroses (P. max)}

Le tableau 1 et la figure 1 présentent les résultats obtenus. Huit $\mathrm{F}_{2}$ monosomiques donnent la même réponse aux 2 souches, parmi elles le $3 \mathrm{~A}$ augmente la résistance, les autres n'ont pas d'effet.

Les $F_{2} 2 B, 3 B$ et $5 B$ qui réduisent $P$. max (augmentation de la résistance) pour la souche 6 , entrainnent une sensibilité accrue avec la souche 42 . Des interactions $F_{2}$ mono $\times$ souches sont mises en évidence (tabl. 3) : prises dans leur ensemble elles concernent surtout les génomes A et D. Le rapprochement du tableau 3 et de la figure 1 montre, en fait, que les $\mathrm{F}_{2}$ à l'origine de cette interaction sont les $\mathrm{F}_{2} 4 \mathrm{~A}, 2 \mathrm{~B}, 3 \mathrm{~B}, 5 \mathrm{~B}, 7 \mathrm{~B}, 1 \mathrm{D}$ et $2 \mathrm{D}$.

\section{Critères de tolérance}

a) Intensité des symptômes sur épis (I.E.)

Aucune $F_{2}$ mono. ne présente à la fois, pour les 2 souches, une réduction ou une augmentation de l'intensité des symptômes sur épis (tabl. 1, fig. 1). Ce sont des $\mathrm{F}_{2}$ totalement différentes qui répondent à chaque souche. Huit $\mathbf{F}_{2}$ monosomiques donnent pour les 2 sou-

\section{TABLEAU 1}

Comparaisons des réponses des $F_{2}$ monosomiques aux deux souches de S. nodorum pour la durée d'incubation (L.P.I.), la vitesse d'extension des nécroses foliaires (P. max) et l'intensité des symptomes sur épis (I.E.).

Comparison of monosomic lines, after inoculation with two strains of Septoria nodorum, for incubation time (L.P.I.), rate of leaf lesion extension (P. max) and symptom intensity on head (I.E.).

\begin{tabular}{|c|c|c|c|c|}
\hline & \multicolumn{3}{|c|}{ Durée d'incubation (L.P.I.) : souche 6} \\
\hline & & 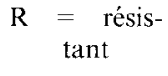 & $\mathbf{E} \underset{\substack{=\\
\text { effet }}}{\text { sans }}$ & $\mathbf{S}=$ sensible \\
\hline \multirow{3}{*}{$\begin{array}{l}\text { L.P.I. souche } \\
42\end{array}$} & $\mathrm{R}$ & $\begin{array}{l}7 \mathrm{~A} \\
2 \mathrm{~B}\end{array}$ & & \\
\hline & $\mathrm{E}$ & $\begin{array}{l}3 A-5 A \\
5 D\end{array}$ & $\begin{array}{l}4 B \\
1 D-2 D-3 D\end{array}$ & $6 \mathrm{D}$ \\
\hline & $\mathrm{S}$ & $\begin{array}{l}4 \mathrm{~A} \\
5 \mathrm{~B}-7 \mathrm{~B}\end{array}$ & $\begin{array}{l}1 \mathrm{~A}-6 \mathrm{~A} \\
3 \mathrm{~B}-6 \mathrm{~B} \\
4 \mathrm{D}-7 \mathrm{D}\end{array}$ & $\begin{array}{l}2 \mathrm{~A} \\
1 \mathrm{~B}\end{array}$ \\
\hline
\end{tabular}

\begin{tabular}{|c|c|c|c|c|}
\hline & & \multicolumn{3}{|c|}{$\begin{array}{c}\text { Vitesse d'extension } \\
\text { des nćcroses (P. max) : souche } 6\end{array}$} \\
\hline & & $\mathrm{R}=$ résis- & $\begin{aligned} & E=\text { sans } \\
& \text { effet }\end{aligned}$ & $\mathrm{S}=$ sensible \\
\hline \multirow{3}{*}{$\begin{array}{l}\text { P. max } \\
\text { souche } 42\end{array}$} & $\mathrm{R}$ & $3 \mathrm{~A}$ & $\begin{array}{l}7 \mathrm{~A} \\
1 \mathrm{D}\end{array}$ & \\
\hline & $\mathrm{E}$ & $2 \mathrm{D}$ & $\begin{array}{l}2 \mathrm{~A}-5 \mathrm{~A} \\
\text { 1B-4B-6B } \\
3 \mathrm{D}-5 \mathrm{D}\end{array}$ & $\begin{array}{l}6 \mathrm{~A} \\
6 \mathrm{D}-7 \mathrm{D}\end{array}$ \\
\hline & $\mathrm{S}$ & 2B-3B-5B & $\begin{array}{l}1 \mathrm{~A}-4 \mathrm{~A} \\
7 \mathrm{~B} \\
4 \mathrm{D}\end{array}$ & \\
\hline
\end{tabular}

\begin{tabular}{|c|c|c|c|c|}
\hline & & \multicolumn{3}{|c|}{$\begin{array}{l}\text { Intensité des symptômes } \\
\text { sur épis (I.E.): souche } 6\end{array}$} \\
\hline & & 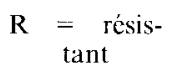 & $\mathbf{E}=\underset{\text { effet }}{=}$ sans & $\mathrm{S}=$ sensible \\
\hline \multirow{3}{*}{$\begin{array}{l}\text { I.E. souche } \\
42\end{array}$} & $\mathrm{R}$ & & $\begin{array}{l}2 \mathrm{~A}-6 \mathrm{~A} \\
5 \mathrm{D}\end{array}$ & \\
\hline & $\mathrm{E}$ & $\begin{array}{l}5 \mathrm{~A} \\
6 \mathrm{~B}-7 \mathrm{~B}\end{array}$ & $\begin{array}{l}7 \mathrm{~A} \\
1 \mathrm{~B}- \\
2 \mathrm{~B}-3 \mathrm{~B}-5 \mathrm{~B} \\
\text { 3D-4D-7D }\end{array}$ & $\begin{array}{l}1 \mathrm{~A}-3 \mathrm{~A} \\
4 \mathrm{~B} \\
1 \mathrm{D}-6 \mathrm{D}\end{array}$ \\
\hline & $\mathrm{S}$ & & $\begin{array}{l}4 \mathrm{~A} \\
2 \mathrm{D}\end{array}$ & \\
\hline
\end{tabular}

ches une réponse identique à celle de la $F_{2}$ disomique. On note des interactions significatives entre souches et $\mathrm{F}_{2}$ monosomiques regroupées par génomes ou par groupes homéologues (tabl. 4). Les $\mathrm{F}_{2}$ les plus impliquées dans cette interaction sont les $2 \mathrm{~A}, 3 \mathrm{~A}, 6 \mathrm{~A}, 2 \mathrm{~B}, 3 \mathrm{~B}$, 


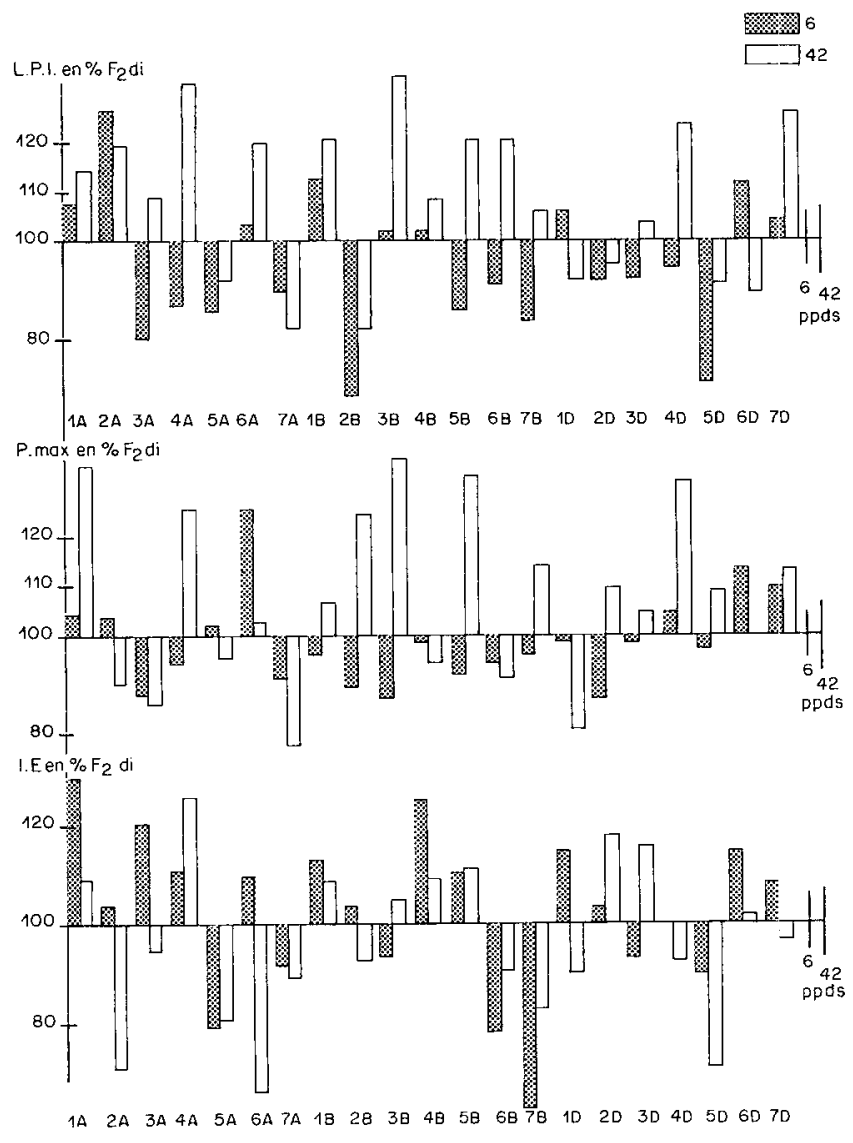

Figure 1

Représentation graphique des réponses des $F_{2}$ monosomiques exprimées en p. 100 de la réponse $F_{2}$ disomique aux souches 6 ou 42 de $\mathrm{S}$. nodorum pour la durée d'incubation (L.P.I.), la vitesse d'extension des nécroses foliaires (P. max) et l'intensité des symptômes sur épis (I.E.). Mise en évidence des interactions $F_{2}$ monosomiques $\times$ souches du parasite.

Graphical illustration of the responses of monosomic lines, as $\%$ of that of the disomic, after inoculation with strains 6 and 42 of Septoria nodorum for: incubation time (L.P.I.), rate of lesion extension $(P . \max )$, and symptom intensity on head (I.E.), showing the inieractions between strains and monosomic lines.
$1 \mathrm{D}$ et $3 \mathrm{D}$; le groupe d'homéologue VII n'intervient pas dans celle-ci.

\section{b) Poids de 1000 grains des plantes malades (P.M.G.M.)}

L'impossibilité de calculer dans cet essai le poids relatif de 1000 grains et la méconnaissance de l'effet de la monosomie, elle-même, sur le poids de 1000 grains sains rendent difficile l'interprétation des résultats. Toutefois, nous savons (RAPILLY et al., 1988) que les monosomiques 1A, 2A, 6A, 3B, 6B, 7B, 2D, 3D, 4D, 7D ont un poids de 1000 grains sains non différent de celui des disomiques. Pour ces familles l'étude du rapport

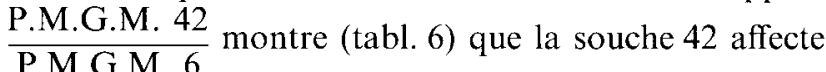
plus les $\mathrm{F}_{2} 1 \mathrm{~A}, 2 \mathrm{~A}, 6 \mathrm{~A}, 6 \mathrm{~B}, 7 \mathrm{~B}, 2 \mathrm{D}$ et $7 \mathrm{D}$ et, pour la souche 6 , les $\mathrm{F}_{2} 3 \mathrm{~B}$ et $4 \mathrm{D}$. Une même réponse est obtenue, aux 2 souches, avec la $F_{2} 3 \mathrm{D}$. Ces différences difficiles à détecter pour les autres $F_{2}$ expliquent les interactions $\mathrm{F}_{2} \times$ souches mises en évidence (tabl. 5) et qui semblent particulièrement importantes pour les $\mathrm{F}_{2}$ monosomiques 1A, 4A, 7A, 2B, 3B, 1D et 4D. La comparaison des classements des $F_{2}$ contaminées avec l'une ou l'autre souche montre (tabl.6) que le P.M.G.M. des $\mathrm{F}_{2} 5 \mathrm{~A}, 4 \mathrm{~B}, 5 \mathrm{D}$ et $6 \mathrm{D}$ est inférieur à celui des disomiques et qu'il faut rajouter à cette liste les $F_{2} 7 \mathrm{~B}$ et $7 \mathrm{D}$ pour la souche 42 et la $\mathrm{F}_{2} 5 \mathrm{~B}$ pour la souche 6 .

\section{Ensemble des paramètres considérés}

Seules les $F_{2} 3 D$ et $6 \mathrm{D}$ donnent, pour les 4 éléments considérés, la même réponse aux 2 souches. La $\mathrm{F}_{2} 3 \mathrm{D}$ ne diffère pas de la $F_{2}$ disomique mais, la $F_{2} 6 \mathrm{D}$ est plus sensible, les 4 éléments pris en compte agissant dans le même sens. Cependant, suivant la souche ou le paramètre considéré, les $\mathrm{F}_{2} 3 \mathrm{~A}$ et $7 \mathrm{~A}$ sont ou plus résistantes ou non différentes de la $F_{2}$ disomique; globalement ces

TABLEAU 2

Mise en évidence pour la durée d'incubation (L.P.I.) des interactions $F_{2}$ monosomiques $x$ souches aux niveaux des génomes et des groupes homéologues. Interactions between monosomic lines and strains for incubation time.

\begin{tabular}{|c|c|c|c|c|c|c|c|c|c|c|c|c|}
\hline \multirow[b]{2}{*}{ Variations } & \multicolumn{3}{|c|}{$\begin{array}{c}\text { Ensemble } \\
\text { des génomes }\end{array}$} & \multicolumn{3}{|c|}{ Génome A } & \multicolumn{3}{|c|}{ Génome B } & \multicolumn{3}{|c|}{ Génome D } \\
\hline & d.d.l. & C.M. & F. & d.d.l. & C.M. & F. & d.d.l. & C.M. & F. & d.d.l. & C.M. & F. \\
\hline Totale & 828 & 27,9 & - & 272 & 27,5 & - & 278 & 27,78 & - & 276 & 28,5 & - \\
\hline Souches & 1 & 6,04 & ns & 1 & 1,1 & $\mathrm{~ns}$ & 1 & 13,44 & ns & 1 & 0,9 & ns \\
\hline $\mathrm{F}_{2}$ & 20 & 68,34 & 2,98 & 6 & 78,4 & 3,79 & 6 & 121 & 5,39 & 6 & 27,55 & ns \\
\hline Erreur & 787 & 22,9 & 一 & 269 & 20,93 & - & 265 & 22,43 & - & 263 & 25,27 & - \\
\hline
\end{tabular}

Valeur du F de l'interaction par groupe homéologue

\begin{tabular}{|c|c|c|c|c|c|c|c|}
\hline & & & & & & & \\
\hline & $\mathrm{I}$ & II & III & IV & $\mathrm{V}$ & VI & VII \\
\hline F avec 2 et 109 à 105 d.d.l. & 10,337 & ns & 11,66 & 16,4 & 10,95 & 8,79 & 20,70 \\
\hline
\end{tabular}


TABLEAU 3

Mise en évidence pour la vitesse d'extension des nécroses foliaires ( $P$. max) des interactions $F_{2}$ monosomiques $x$ souches aux niveaux des génomes et des groupes homéologues.

Interactions between monosomic lines and strains for rate of leaf lesion extension (P. max).

\begin{tabular}{|c|c|c|c|c|c|c|c|c|c|c|c|c|}
\hline \multirow[b]{2}{*}{ Variations } & \multicolumn{3}{|c|}{$\begin{array}{c}\text { Ensemble } \\
\text { des génomes }\end{array}$} & \multicolumn{3}{|c|}{ Génome A } & \multicolumn{3}{|c|}{ Génome B } & \multicolumn{3}{|c|}{ Génome D } \\
\hline & d.d.l. & C.M. & F. & d.d.l. & C.M. & F. & d.d.l. & C.M. & $\mathrm{F}$. & d.d.l. & C.M. & $\mathrm{F}$. \\
\hline Totale & 811 & 1,03 & - & 267 & 0,984 & - & 268 & 1,03 & - & 274 & 1,0674 & - \\
\hline Souches & 1 & 15,163 & 213,5 & 1 & 0,774 & ns & 1 & 30,18 & 32,5 & 1 & 0,175 & ns \\
\hline $\mathrm{F}_{2}$ & 20 & 4,098 & 57,7 & 6 & 10,8 & 48,6 & 6 & 0,5 & ns & 6 & 1,405 & 2,36 \\
\hline Erreur & 770 & 0,071 & - & 254 & 0,222 & - & 261 & 0,93 & - & 261 & 0,595 & - \\
\hline
\end{tabular}

Interactions par groupe homéologues

\begin{tabular}{lccccccc}
\cline { 2 - 7 } & I & II & III & IV & V & VI & VII \\
\hline F avec 2 et 109 à 105 d.d.l. & 252 & ns & 369 & ns & ns & ns & 14,18 \\
\hline
\end{tabular}

TABLEAU 4

Mise en évidence pour l'intensité des symptomes sur épis (I.E.) des interactions $F_{2}$ monosomiques x souches aux niveaux des génomes et des groupes homéologues.

Interactions between monosomic lines and strains for symptom intensity on the head.

\begin{tabular}{|c|c|c|c|c|c|c|c|c|c|c|c|c|}
\hline \multirow{3}{*}{ Variations } & \multicolumn{3}{|c|}{$\begin{array}{l}\text { Ensemble } \\
\text { des génomes }\end{array}$} & \multicolumn{3}{|c|}{ Génome A } & \multicolumn{3}{|c|}{ Génome B } & \multicolumn{3}{|c|}{ Génome D } \\
\hline & d.d.1. & C.M. & F. & d.d.l. & C.M. & F. & d.d.l. & C.M. & $\mathrm{F}$. & d.d.1. & C.M. & $\mathrm{F}$. \\
\hline & 834 & 1,053 & - & 274 & 1,082 & - & 279 & 1,075 & - & 279 & 0,996 & - \\
\hline Souches & 1 & 0,064 & ns & 1 & 0,244 & ns & 1 & 1.564 & 3,83 & 1 & 0,007 & ns \\
\hline $\mathrm{F}_{2}$ & 20 & 6,1225 & 15,66 & 6 & 8,928 & 32,1 & 6 & 8.71 & 21,34 & 6 & 2,13 & 4,39 \\
\hline Interaction & 20 & 22,276 & 56,97 & 6 & 28,739 & 105,52 & 6 & 22,95 & 56,24 & 6 & 22,69 & 46,78 \\
\hline Erreur & 793 & 0,391 & - & 261 & 0,2776 & - & 266 & 0,408 & - & 266 & 0,485 & - \\
\hline
\end{tabular}

Interaction par groupes homéologues

\begin{tabular}{lccccccc} 
& I & II & III & IV & V & VI & VII \\
\hline F avec 2 et 109 à 105 d.d.1. & 11,84 & 36,24 & 357 & 14,9 & 22,44 & 22,82 & ns \\
\hline \hline
\end{tabular}

chromosomes améliorent donc la résistance et la tolérance aux 2 souches du parasite. Par contre, les $\mathrm{F}_{2} 4 \mathrm{~B}$ et 6B se montrent sans effet ou plus sensibles et ont donc une action globale négative. Il en est de même pour les $1 \mathrm{~A}$ et $4 \mathrm{D}$ pour 3 éléments sur 4 . Les autres $F_{2}$ se distinguent, de façon différente, selon la souche ou le caractère considéré ; leur effet est donc, au vu de ces données, difficile à prévoir globalement.

Le tableau 7 indique, pour tous les caractères mesurés, les chromosomes qui apparaissent les plus impliqués dans les interactions souches $\times F_{2}$ monosomique. Les
3 génomes $\mathrm{A}, \mathrm{B}, \mathrm{D}$ apparaissent concernés; seuls les chromosomes 5A, 1B, 4B, 5D et 7D ne sont pas apparus impliqués dans ces dernières.

\section{DISCUSSION}

NELSON (1978), a associé la résistance horizontale, telle que l'a définie Van Der Plank (1963), aux résistances partielles. Toutes deux ont pour effet de réduire 
TABLEAU 5

Analyses de variances effectuées sur le poids de l 000 grains malades (P.M.G.M.) pour comparer les souches 6 et 42 Analysis of variance for P.M.G.M. in comparisons between strains 6 and 42.

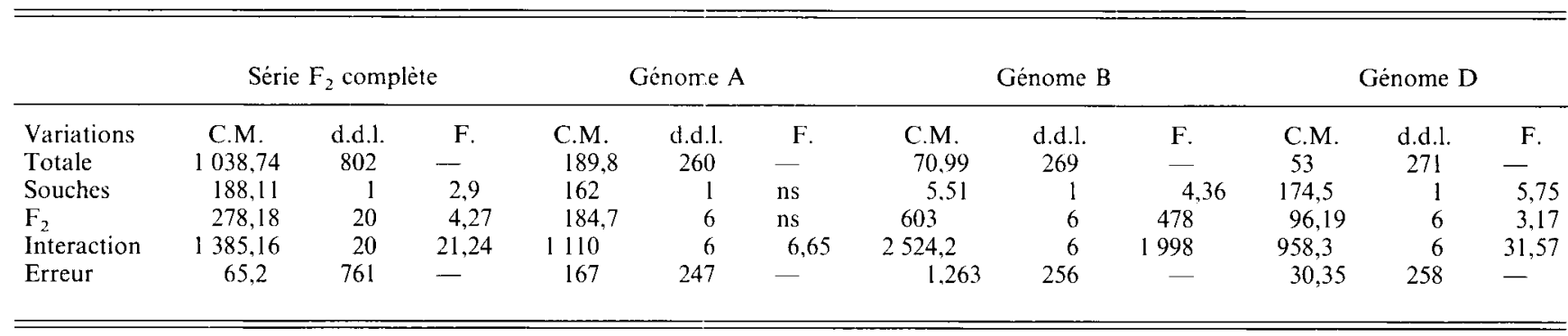

Interactions monosomiques $\mathrm{x}$ souches par groupe d'homéologie

\begin{tabular}{|c|c|c|c|c|c|c|c|}
\hline \multirow{2}{*}{ Valeur du F avec 2 et 109 à 115 d.d.l. monosomique $x$ souche } & I & II & III & IV & $\mathrm{V}$ & VI & VII \\
\hline & 134,2 & 25,8 & ns & 58,6 & ns & $\mathrm{ns}$ & 32,6 \\
\hline
\end{tabular}

TABLEAU 6

Classement des $F_{2}$ monosomiques pour le P.M.G.M. exprimé en p. 100 des $F_{2}$ disomiques contaminées par la souche 6 ou la souche 42 et valeur du rapport

\section{$\underline{\text { P.M.G.M. } 42} \times \frac{\text { P.M.G.M.F } 2 \text { di } 42}{\text { P.M.G.M. }}$}

Classification for P.M.G.M. of monosomic lines after inoculation at flowering stage with strains 6 or 42.

\begin{tabular}{|c|c|c|}
\hline $\begin{array}{l}\text { P.M.G.M. } \\
\text { souche } 6\end{array}$ & $\begin{array}{l}\text { P.M.G.M. } \\
\text { souche } 42\end{array}$ & $\frac{\text { P.M.G.M. } 42}{\text { P.M.G.M. } 6}$ \\
\hline $4 B=81,31$ & $4 \mathrm{~B}=80.51$ & $1 \mathrm{~A}=0,84$ \\
\hline$\overline{5 \mathrm{~A}}=84,34$ & $\overline{6 \mathrm{D}}=82,02$ & $1 \mathrm{~B}==0,84$ \\
\hline$\overline{5 B}=88,17$ & $\overline{5 \mathrm{D}}=83,03$ & $2 \mathrm{D}=0.0,85$ \\
\hline$\overline{6 \mathrm{D}}=89,34$ & $\overline{7 \mathrm{D}}=84.63$ & $7 \mathrm{~A}=0.86$ \\
\hline$\overline{5 \mathrm{D}}=92,10$ & $\overline{7 B}=86,14$ & $7 \mathrm{D}=0.86$ \\
\hline$\overline{4 \mathrm{D}}=92,94$ & $\overline{5 \mathrm{~A}}=88,15$ & $4 \mathrm{~A}=0,86$ \\
\hline $7 \mathrm{~B}=93,38$ & $\overline{7 \mathrm{~A}}=89,19$ & $5 \mathrm{D}=0,86$ \\
\hline $2 \mathrm{~B}=94,09$ & $1 \mathrm{~A}=89,4$ & $3 \mathrm{~A}=0,87$ \\
\hline $7 \mathrm{D}=94.5$ & $1 D=91,03$ & $1 \mathrm{D}=0.88$ \\
\hline $2 \mathrm{~A}=95,23$ & $2 \bar{D}=91.61$ & $6 \mathrm{~B}=0.88$ \\
\hline $3 \mathrm{D}=95,8$ & $6 \mathrm{~A}=92,67$ & $6 \mathrm{D}=0,88$ \\
\hline $6 \mathrm{~A}=97,95$ & $2 \mathrm{~A}=94,33$ & $7 \mathrm{~B}=0,88$ \\
\hline $7 A=99,17$ & $4 \mathrm{~A}=94.38$ & $6 \mathrm{~A}=0,90$ \\
\hline $1 D=99,2$ & $6 B=94,44$ & $2 \mathrm{~A}=0,95$ \\
\hline $1 \mathrm{~A}=101,25$ & $3 \mathrm{D}=96.29$ & $4 \mathrm{~B}=0,95$ \\
\hline $6 \mathrm{~B}=102,1$ & $5 B=96.58$ & $3 \mathrm{D}:=0,96$ \\
\hline $2 \mathrm{D}=102,45$ & $3 \mathrm{~A}=96,84$ & $2 B=0,99$ \\
\hline $3 B=103,17$ & $2 B=97.67$ & $5 \mathrm{~A}=1.0$ \\
\hline $4 \mathrm{~A}=105.49$ & $1 B=98.14$ & $3 \mathrm{~B}=1.01$ \\
\hline $3 A=106$ & $4 D=98.88$ & $4 \mathrm{D}=1.02$ \\
\hline $1 \mathrm{~B}=111,11$ & $3 B=109.02$ & $5 \mathrm{~B}-1.05$ \\
\hline $100=36 \mathrm{~g}, 91$ & $100=38 \mathrm{~g}, 59$ & \\
\hline
\end{tabular}

— différents au seuil de 5 p. 100 de la $F_{2}$ disomique.

la progression des épidémies. Cette assimilation est admissible du point de vue épidémiologique. Les résultats rapportés ci-dessus permettent de généraliser certaines conclusions déjà formulées (AuRIAU et al., 1988; RAPILLY et al., 1988) ; en effet, il ressort très nettement que :
1. Les éléments constitutifs de la résistance partielle foliaire à la septoriose ont un déterminisme génétique complexe. Le nombre de chromosomes impliqué varie avec le paramètre pris en compte pour apprécier la résistance, et avec la souche du parasite : de 4 à 8 chromosomes pour la souche 6 , de 2 à 3 pour la souche 42 . Les gènes actifs sur les paramètres L.P.I. et P. max peuvent être portés par le même chromosome (cas des $3 \mathrm{~A}, 2 \mathrm{~B}, 5 \mathrm{~B}$ et $6 \mathrm{D}$ pour la souche 6 , des $1 \mathrm{~A}, 4 \mathrm{~A}, 7 \mathrm{~A}, 5 \mathrm{~B}$, $7 \mathrm{~B}$ et $4 \mathrm{D}$ pour la souche 42 ). Mais certains chromosomes ne sont actifs que pour l'un de ces deux paramètres (par exemple 7A, 1B, 2D, 7D pour la souche 6;1B, 1D, 2D pour la souche 42 ). Cette complexité est accrue par le fait que pour un paramètre, un même chromosome peut porter un gène de résistance vis-à-vis d'une souche et un gène de sensibilité pour l'autre souche (cas des $2 \mathrm{~B}$, 3B, 5B pour P. max, 4A, 5B, 7B pour la L.P.I.). On peut aussi émettre l'hypothèse qu'un même gène conduit à des effets différents suivant la souche pathogène, ce qui révèlerait des interactions spécifiques.

Pour évaluer le poids de divers éléments constitutifs des résistances partielles LEONARD \& MUNDT (1984), RAPILLY (1977) lient, dans leurs simulations biométriques, les durées d'incubation et de latence avec l'extension des surfaces sporulantes donc contagieuses. Cette liaison souvent constatée, nous l'observons encore dans cette étude, où les coefficients de corrélation entre L.P.I. et P. max sont de $0,42 \mathrm{~S}$. pour la souche 6 et de $0,6 \mathrm{H}$.S. pour la souche 42 , est due à quelques chromosomes et n'est que partielle. Elle n'est pas généralisable sur le plan des mécanismes héréditaires comme le montrent les réponses du chromosome $2 \mathrm{~B}$.

2. Les deux critères retenus pour juger de la tolérance ont aussi un déterminisme génétique complexe, ce que Kleijer et al. (1980) avait déjà montré pour le poids relatif de 1000 grains. Il apparaît, de plus, que I.E. et P.M.G.M. sont, d'une part, indépendants entre eux et, d'autre part, indépendants des mécanismes qui régissent la résistance partielle foliaire. Les coefficients de corrélation calculés entre P. max et I.E. varient, suivant la 
TABLEAU 7

Chromosomes les plus impliqués dans l'interaction entre les paramètres de résistance partielle ou de tolérance et les souches 6 et 42 de Septoria nodorum.

Implication of chromosomes in interactions for partial resistance or tolerance with the two strains of Septoria nodorum.

\begin{tabular}{|c|c|c|c|c|c|c|c|c|c|c|c|c|c|c|c|c|c|c|c|c|}
\hline \multirow{2}{*}{ Paramètres } & \multicolumn{20}{|c|}{ Dénomination des chromosomes } \\
\hline & $1 \mathrm{~A}$ & $2 \mathrm{~A}$ & $3 \mathrm{~A}$ & $4 \mathrm{~A}$ & $5 \mathrm{~A}$ & $6 \mathrm{~A}$ & $7 \mathrm{~A}$ & $2 \mathrm{~B}$ & $3 \mathrm{~B}$ & 4B & $5 B$ & $6 \mathrm{~B}$ & $7 \mathrm{~B}$ & $1 \mathrm{D}$ & $2 \mathrm{D}$ & $3 \mathrm{D}$ & $4 \mathrm{D}$ & $5 \mathrm{D}$ & $6 \mathrm{D}$ & $7 \mathrm{D}$ \\
\hline L.P.I. & & & $\mathrm{x}$ & $\mathrm{x}$ & & & & & & & $\mathrm{X}$ & $\mathrm{X}$ & $\mathrm{X}$ & & & & $\mathrm{x}$ & & $\mathrm{X}$ & \\
\hline P. max & & & & $\mathrm{X}$ & & & & $\mathrm{x}$ & $\mathrm{X}$ & & $\mathrm{X}$ & & $\mathrm{X}$ & $\mathrm{X}$ & $\mathrm{x}$ & & & & & \\
\hline I.E. & & $\mathrm{X}$ & $\mathrm{X}$ & & & $\mathrm{X}$ & & $\mathrm{X}$ & $\mathrm{X}$ & & & & & $\mathrm{X}$ & & $\mathrm{X}$ & & & & \\
\hline P.M.G.M. & $\mathrm{X}$ & & & $\mathrm{X}$ & & & $\mathrm{X}$ & $\mathrm{X}$ & & & $\mathrm{X}$ & & & $\mathrm{X}$ & & & $\mathrm{X}$ & & & \\
\hline
\end{tabular}

$\mathrm{X}=$ Chromosomes impliqués dans une interaction

souche, de 0,13 à 0,29 et ne sont pas significatifs. Sauf pour le chromosome $6 \mathrm{D}$ les gènes qui gouvernent l'I.E. et le P.M.G.M. semblent indépendants entre eux. Ainsi le $3 \mathrm{~A}$ porte des gènes qui accroissent l'I.E. sans entraîner de réduction du poids de 1000 grains des plantes malades ce qui peut être consécutif à la réduction de la maladie qu'il provoque sur feuille. Les variations du P.M.G.M., enregistrées suivant les $F_{2}$ monosomiques pourraient être dues à la conjonction de deux actions, d'une part, celui du parasite et d'autre part celle due à l'état monosomique. Mais l'étude du

rapport $\frac{\text { P.M.G.M. souche } 42}{\text { P.M.G.M. souche } 6}$ pour les $F_{2}$ mono. ( $1 \mathrm{~A}$,

2A, 6A, 3B, 6B, 7B, 2D, 4D, 5D, 7D) dont le poids des grains ne diffère pas de celui de la $F_{2}$ disomique (RAPILlY et al., 1988) confirme bien que le P.M.G.M. est éminemment variable suivant la souche et sans liaison avec l'expression des symptômes sur épis. Des inversions de réponse en fonction du stade de développement ou de l'organe considéré ont déjà été signalées chez les céréales. Elles sont bien connues chez le blé en fonction du stade, pour la résistance aux parasites obligatoires (LUPTON \& JOHNSON, 1970); elles le sont aussi entre la feuille de riz et la panicule, pour la résistance à la pyriculariose (KIYOSAWA, 1982).

La reconnaissance d'une relation, entre l'hôte et le parasite, de virulence ou d'agressivité est plus à rechercher dans le nombre de gènes impliqués que dans la signification biologique (ici une relation de spécificité) d'un calcul statistique. Les effets finaux que ces gènes induisent sont très importants à apprécier. Si la manifestation visuelle de ces derniers est l'impossibilité du développement de la maladie (avant ou après pénétration) ils correspondent à une notion qualitative. Si un développement, plus ou moins abondant, de la maladie est possible, ils correspondent à une notion quantitative.

Pour la septoriose nous sommes dans le cas où la maladie se manifeste toujours. Cette manifestation finale n'exclut pas qu'à des niveaux plus fins de jugement que ceux utilisés pour apprécier les éléments constitutifs de la résistance partielle, existent des relations gènes pour gènes dans un contexte très polygénique. L'addition de celles-ci n'interdit jamais la maladie mais en module la quantité. Parlevliet (1977) a signalé, pour la rouille naine de l'orge, que des interactions pouvaient être mises en évidence pour la durée de latence entre hôtes et isolats du parasite. Il suggère que la période de latence relève d'un système polygénique, chez l'hôte, qui peut opérer sur une base gène pour gène avec un système polygénique du pouvoir pathogène.

Pour les éléments étudiés ici nous sommes dans un cas similaire avec, chez l'hôte, des gènes de résistance à effet quantitatif et partiel face à des gènes qui, chez le parasite, leur sont complémentaires au sens donné par FLOR (1956). Les interactions constatées entre $F_{2}$ mono. et souches permettent de penser que la véritable dénomination de ces gènes complémentaires est "gènes de résistance partielle spécifique "; il y aurait donc une continuité entre virulence (= qualité) et agressivité (= quantité).

Dans ce cas, ces résistances pourraient être à l'origine de pressions sélectives sur la population parasitaire comme le signalent BEyE \& LAFAY (1988a) pour la résistance générale de la tomate à la verticilliose. Une dérive progressive des populations que sont les souches de $S$. nodorum, vers la spécialisation serait à craindre. Toutefois, en raison du grand nombre de gènes impliqués et de l'homéostase génétique qui favorise les intermédiaires, l'effondrement complet des résistances partielles est difficile à concevoir.

Il faut souligner que les souches 6 et 42 utilisées dans cette étude sont des populations et que chacune d'elle est composée de divers génotypes dont les fréquences respectives déterminent des pouvoirs pathogènes très différents même entre spores prélevées dans une même pycnide (SKaJENNIKOFF, RaPILlY, données non publiées). On peut penser que l'hôte nouveau qu'est le triticale, pour le $S$. nodorum, a sélectionné, au sein des isolats provenant du blé, les constituants qui lui sont le plus adaptés. Dans des expérimentations, sur triticale (SkAJENNIKOFF \& RAPILLY, 1985, et données non publiées), ont montré qu'au sein de la population 6 pouvaient être "prélevés ", par un triticale, des éléments qui lui étaient adaptés et étaient plus pathogènes, pour cet hôte, que l'isolat de départ. Les souches 6 et 42 tendraient, dans ce cas, à être l'équivalent de formes spéciales l'une de type blé l'autre de type triticale. Il faut signaler que OsBOURN et al. (1986) ont montré que les souches, de ce parasite, isolées de blé ou d'orge étaient en fait des isolats spécialisés incapables de s'associer pour former des hétérocaryons. On peut s'interroger pour savoir si, au sein d'une forme spéciale, des souches aptes à surmonter tel ou tel gène de résistance partielle peuvent se différencier comme le suggèrent les travaux de BEYE \& LAFAY (1988b) sur le couple Verticillium dahliae-tomate. 
Une autre hypothèse peut être formulée pour comprendre les interactions constatées: elles peuvent être la simple conséquence du fait que la lignée L22, dont les chromosomes sont tour à tour présents dans les $\mathrm{F}_{2}$ monosomiques, a été sélectionnée pour sa résistance foliaire à un isolat équivalent à la souche 6 , ceci peut expliquer le faible nombre de chromosomes donnant une réponse de résistance foliaire vis-à-vis de la souche 42 et le grand nombre de chromosomes qui, avec la souche 6 , donnent pour l'épi une réponse de sensibilité.

\section{CONCLUSIONS}

Malgré le nombre de gènes impliqués et les interactions constatées, cette étude nous amène à penser que, résistance partielle ou horizontale et résistance verticale, ne diffèrent pas fondamentalement sur le plan des mécanismes génétiques mis en jeu. Par contre, ils aboutissent à des effets finaux qui eux sont totalement différents pour le sélectionneur. Ainsi, pour la souche 6 de $S$. nodorum, en supposant que les 10 chromosomes impliqués dans la résistance des feuilles ne portent chacun qu'un gène, la probabilité d'obtenir, en deuxième génération, une plante homozygote pour ces

10 gènes est de $\frac{1}{1048576}$ (pour la souche 42 avec 4 gènes elle est de $\frac{1}{256}$ ). De plus il serait très difficile de distinguer, pour la résistance, une plante homozygote d'une plante hétérozygote qui serait, elle aussi, partiellement résistante.

Enfin L.P.I. et P. max sont, en partie, indépendants et gouvernés par des gènes récessifs et des gènes dominants (RAPILLY et al., 1988) qui parfois sont portés par le même chromosome.

Toutes ces difficultés conduisent à penser que la sélection pour la résistance doit se conduire à un stade suffisant d'homozygotie en $F_{3}$ et $F_{4}$ et non sur des plantes individuelles mais en jugeant du comportement, homogène ou non, des familles. C'est d'ailleurs un schéma similaire qui nous a permis d'obtenir la lignée L22 (RAPILly et al., 1984). Toutefois, la difficulté pour le sélectionneur est, au cours des divers cycles, de ne pas éroder la résistance en ne retenant que les gènes aux effets les plus marqués et non ceux qui individuellement, ont peu d'action mais combinés à d'autres, ont des effets additifs voir multiplicatifs.

La combinaison des données de test en terrine, sur plantules, pour la L.P.I., de test sur feuilles en survie pour P. max et de contrôles en microparcelles pour juger de l'épidémie doit permettre d'éviter ce risque de dérive sous réserve qu'ils soient compatibles avec la charge de travail du sélectionneur. La méthodologie proposée par RoBINSON (1976) pour la résistance horizontale reposant sur plus d'une dizaine de croisements au hasard, (ce qui est proche de la sélection récurrente), supportant une pression de sélection progressive ne nous paraît possible que pour créer une population source. Celle que nous proposons nous semble adaptée aux finalités des sélectionneurs car elle permet, dans les premiers cycles de sélection, de trier pour les caractères agronomiques en l'absence de septoriose.

Pratiquement il nous paraît évident qu'il faut chercher à associer résistance partielle et tolérance; on ne sait, à ce jour, quelle est la voie la plus efficace mais elles peuvent être complémentaires. Cette association peut se faire, d'une part, lors de la détection de lignées intéressantes, pour la création d'une population source, et d'autre part, en cours de sélection par dédoublement des microparcelles destinées à juger de l'épidémie.

Une dernière question se pose au vu des réponses obtenues avec les 2 souches: faut-il inoculer avec un mélange de souche? Notre réponse est négative car l'agressivité d'une souche et le comportement d'une variété ne peuvent s'apprécier que par de nombreuses notations répétées dans le temps et des observations peu fréquentes ne permettent pas d'associer à une souche l'évolution du taux final de maladie observée. Il faut sélectionner vis-à-vis de la même population et opérer, un cycle sur deux, des contrôles avec des souches isolées d'hôtes très différents. Ces contrôles peuvent se faire, sur familles avancées, en hiver, sur feuilles détachées afin d'exploiter la relation partielle qui existe entre la L.P.I. et P. max.

Cette précaution est nécessaire car Septoria nodorum apparaît comme un parasite «primitif » qui a peu subi de pression de sélection; il présente une grande étendue de variation tant au niveau de sa forme de reproduction asexuée qu'au niveau des ascospores qui concourent à initier les épidémies.

Reçu le 16 mars 1988. Accepté le 2 juillet 1988.

\section{REMERCIEMENTS}

Nous remercions Mmes CaUderon et Rousel dont le laboratoire de cytogénétique est à l'origine de la création du matériel monosomique.

Les remarques de Mrs Chevaugeon et Clerjeau nous ont été très utiles à la réalisation de ce manuscrit.

\section{RÉFÉRENCES BIBLIOGRAPHIQUES}

\begin{abstract}
Auriau P., Rapilly F., Cauderon Y., 1988. Analyse monosomique de la résistance à Septoria nodorum Berk. chez le blé tendre (Triticum aestivum L. em Thell. (Will) M.K.). Agronomie, 8 (1), 71-77.

Beye I., Lafay F., 1988a. Verticilliose de la tomate: Analyse des interactions entre l'agressivité de l'agent pathogène et la résistance générale de l'hôte. Agronomie, 8 (4), 351-361.

Beye I., Lafay F., 1988b. Verticilliose de la tomate : Conséquences des interactions entre l'agressivité de l'agent pathogène et la résistance de l'hôte au niveau des populations. Agronomie, 8 (5), 435-439.
\end{abstract}

Chapman V., Riley R., 1966. The allocation of the chromosomes of
T. aestivum to the $\mathrm{A}$ and $\mathrm{B}$ genomes and evidence of the genome structure. Can. J. Genet. Cytol., 8, 57-63.

Flor H. H., 1956. The complementary genic systems in flax and flax rust. $A d v$. Genet., 8, 29-54.

Kiyosawa S., 1982. Genetics and epidemiological modeling of breakdown of plant disease resistance. Ann. Rev. Phytopathol., 20, 93-117.

Kleijer G., Fossati A., Paccaud F. X., 1980. Genetic analysis of tolerance to Septoria nodorum Berk. in wheat using chromosome substitution lines. Z. Pfanzenzüchtung, 85, 227-293. 
Leonard K. J., Mundt C. C., 1984. Methods for estimating epidemiological effects of quantitative resistance to plant diseases. J. theor. Appl. Gen., 67, 219-230.

Lupton P. C., Johnson H. R., 1970. Breeding for mature-plant resistance to yellow rust in wheat. Ann. appl. Biol., 66, 137-J43.

Nelson R. R., 1978. Genetics of horizontal resistance to plant diseases. Ann. Rev. Phytopathol., 16, 359-378.

Osbourn A. E., Scott R. R., Caten C. E., 1986. The effects of host passaging on the adaptation of Septoria nodorum to wheat or barley. Plant Pathol., 35, 135-145.

Parlevliet J. E., 1977. Evidence of differential interaction in the polygenic Hordeum vulgare-Puccinia hordei relation during epidemic development. Phytopathology, 67, 776-778.

Rapilly F., 1977. Recherche de facteurs de résistance horizontale à la septoriose du blé (Septoria nodorum Berk.). Ann. Phytopathol., 9, 1-19. Rapilly F., 1984. Réflexions sur la résistance des plantes aux attaques parasitaires. Sélection. fr., 34, 5-18.
Rapilly F., Auriau P., Laborie Y., Depatureaux C., 1984. Recherches sur la résistance partielle du blé tendre à Septoria nodorum Berk. Agronomie, 4 (7), 639-651.

Rapilly F., Auriau P., Cauderon Y., Richard H., Depatureaux C., 1988. Réactions à la contamination par Leptosphaeria nodorum Mull. des feuilles, épis et grains de plantes $\mathrm{F}_{2}$ issues du croisement entre une lignée de blé résistante et la série monosomique de Courtot. Agronomie, 8, (1), 647-652.

Robinson R. A., 1976. Plant pathosystems. Springer Verlag 184 p. Skajennikoff M., Rapilly F., 1985. Consequences of Triticale introduction on the parasites fungi of wheat. $3^{\text {rd }}$ Eurocarpia cereal meeting "Genetic and breeding of triticale". 2-5 July 1984. Publ. I.N.R.A. Paris, p. 349-357.

Trottet M., Merien P., 1982. Analyse du comportement de vingt lignées de blé tendre vis-à-vis de Septoria nodorum Berk. Agronomie, $2(8), 727-734$.

Van Der Plank J. E., 1963. Plant diseases: epidemics and controt. Acad. Press., New York, London, 349 pp. 